\title{
Continuous measurement of global difference coupling using a phase-locked-loop tune meter in the Relativistic Heavy Ion Collider
}

\author{
Y. Luo, ${ }^{*}$ P. Cameron, A. Dellapenna, L. Hoff, A. Marusic, S. Peggs, and C. Schultheiss \\ Brookhaven National Laboratory, Upton, New York 11973, USA \\ R. Jones \\ European Organization for Nuclear Research (CERN), Geneva, Switzerland \\ (Received 9 June 2006; published 14 December 2006)
}

\begin{abstract}
We present a new technique to continuously measure and compensate the global difference coupling coefficient through the continuous measurements of eigenmode projection parameters, using a high resolution phase-locked-loop tune meter. First, four eigenmode projection parameters are defined as the observables for weak difference coupling. Then, their analytical expressions are obtained using the strict matrix treatment and the Hamiltonian perturbation theory of linear coupling. From these parameters, the complex global coupling coefficient can be fully determined and compensated. This method was successfully demonstrated in the Relativistic Heavy Ion Collider (RHIC) 2006 run.
\end{abstract}

\section{INTRODUCTION}

The skew quadrupole strength scan is the conventional method to measure and correct global coupling [1-5]. However, it is unsuitable for coupling measurements and corrections during ramping. As a logical extension, the skew quadrupole modulation technique was proposed to deal with global decoupling on the ramp, with modulation of either coupling amplitude or coupling angle [6-9]. To track the fast tune changes, a phase-locked-loop (PLL) tune meter [10-13] was used.

In the previously employed above methods for coupling measurements and corrections, the fractional eigentune split is used as the observable. This has been proved to be less sensitive when the accelerator is working close to the difference coupling resonance line. To detect the minimum of the fractional eigentune split, additional coupling sources must be introduced into the accelerator. All of the above methods are used in feed-forward mode. In applying tune feedback without decoupling feedback on the RHIC ramp, we sometimes experienced a mix-up of PLL eigentune tracking under the coupled situation $[7,8]$. To solve this problem, thereby ensuring a robust PLL tune measurement system, continuous coupling measurements and corrections are needed $[14,15]$.
To improve coupling measurements, during the RHIC 2004 run, the PLL was reconfigured to measure the projections of both eigenmodes onto $x$ and $y$ axes. In addition, a formalism was developed to properly parametrize this measurement $[15,16]$. According to this formalism, the global coupling coefficient can be determined from the measurements of the eigenmode projection parameters.

In the following, we first define four eigenmode projection parameters for the coupled betatron motion. Then, their analytical expressions are obtained via both the strict matrix treatment and the Hamiltonian perturbation theory. From the measurements of these parameters, the complex global coupling coefficient can be fully determined and compensated. This method was successfully demonstrated in the RHIC 2006 run. The continuous coupling measurement yielded a global decoupling feedback along with a robust tune feedback.

\section{EIGENMODE PROJECTION PARAMETERS}

\section{A. Definitions}

At one observation point in the ring, the beam center's $(x, y)$ coordinates of a free oscillation motion or of a coherently excited motion are given by

$$
\left\{\begin{array}{l}
x_{n}=A_{I, x} \cos \left[2 \pi Q_{I}(n-1)+\phi_{I, x}\right]+A_{I I, x} \cos \left[2 \pi Q_{I I}(n-1)+\phi_{I I, x}\right] \\
y_{n}=A_{I, y} \cos \left[2 \pi Q_{I}(n-1)+\phi_{I, y}\right]+A_{I I, y} \cos \left[2 \pi Q_{I I}(n-1)+\phi_{I I, y}\right]
\end{array}\right.
$$

\footnotetext{
*Electronic address: yluo@bnl.gov
} 
where $Q_{I}$ and $Q_{I I}$ are the eigentunes with coupling sources. $A_{i, z}$ and $\phi_{i, z}, i=I, I I, z=x, y$, are the eigenmode $i$ 's projection amplitude and projection phase onto $z$ axis. $A_{i, z}$ are non-negative numbers.

Besides the fractional eigentune split, we define four eigenmode projection parameters as the coupling observables. $R_{I}$ and $R_{I I}$ are the ratios of eigenmode projection amplitudes between the horizontal and vertical projections,

$$
\begin{gathered}
R_{I}=\frac{A_{I, y}}{A_{I, x}}, \\
R_{I I}=\frac{A_{I I, x}}{A_{I I, y}} .
\end{gathered}
$$

$\Delta \phi_{I}$ and $\Delta \phi_{I I}$ are the eigenmode projection phase differences between the horizontal and vertical projections,

$$
\begin{gathered}
\Delta \phi_{I}=\phi_{I, y}-\phi_{I, x}, \\
\Delta \phi_{I I}=\phi_{I I, x}-\phi_{I I, y} .
\end{gathered}
$$

Here, we assumed that eigenmodes $I$ and $I I$ are related to the horizontal and vertical planes, respectively. Therefore, if there is no coupling in the accelerator, $R_{I}=R_{I I}=0$.

\section{B. Simulation}

Before giving the analytical expressions to these eigenmode projection parameters, we carry out numerical simulations to check their behavior in a 2D decoupling scan, using a simplified accelerator model of the RHIC's linear optics.

The uncoupled tunes are set to $\left(Q_{x, 0}, Q_{y, 0}\right)=$ $(28.22,29.23)$. Three thin-lens skew quadrupoles are equidistantly inserted into the ring model. The first skew quadrupole's strength is set to $\left(k_{s} d l\right)_{1}=0.005 \mathrm{~m}^{-1}$ to introduce the coupling. The other two skew quadrupoles are used for scanning. Based on the optics model, the global coupling correction strengths are $\left(k_{s} d l\right)_{2,3}=0.005 \mathrm{~m}^{-1}$.

Figure 1 shows the eigentune splits in the $2 \mathrm{D}$ scan, and Fig. 2 shows the projection amplitude ratios $R_{I, I I}$. The projection ratios are much more sensitive than the eigentune split while scanning close to the globally uncoupled point.

Figure 3 shows the projection phase difference $\Delta \phi_{I}$, where $\Delta \phi_{I}$ remains constant if the direction of the decoupling scan points toward the globally uncoupled point. We also found that there is a sudden $\pi$ phase jump in $\Delta \phi_{I}$ when the scanning crosses the uncoupled point. In the following, we will show that $\Delta \phi_{I}$ actually is the phase of the global difference coupling coefficient $C^{-}$.

More detailed simulations are given in [16-18]. In [17], comparisons of the projection parameters between three different approaches, together with the numerical simulations, are presented.

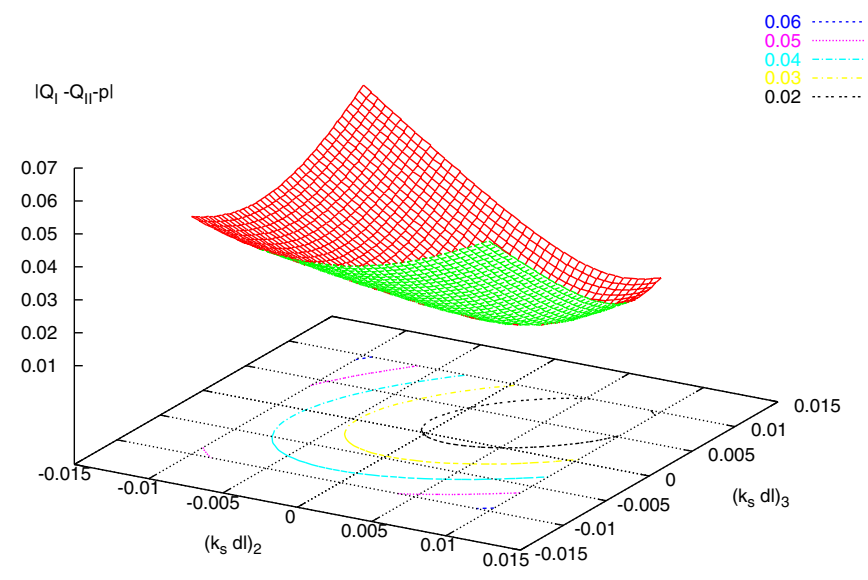

FIG. 1. (Color) Eigentune split in a 2D decoupling scan.

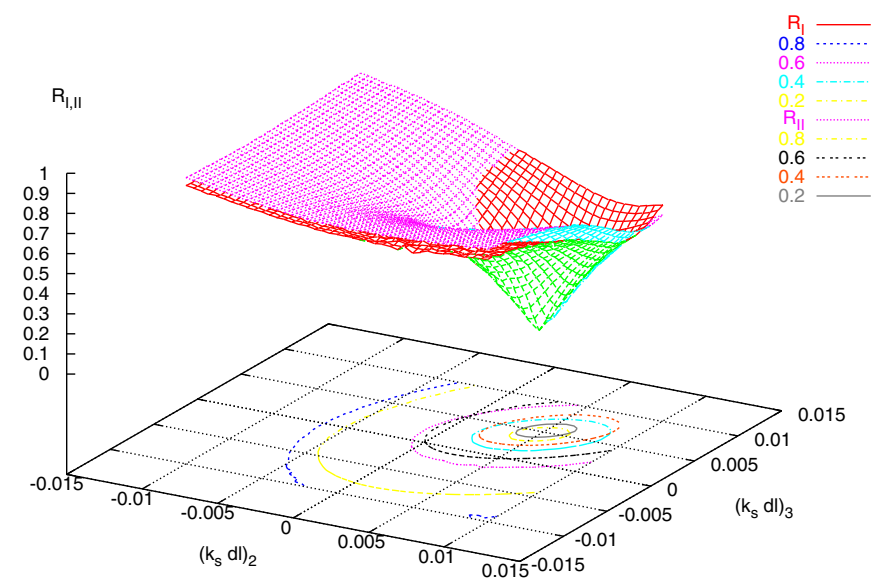

FIG. 2. (Color) Projection amplitude ratios $R_{I I I I}$ in a 2D decoupling scan.

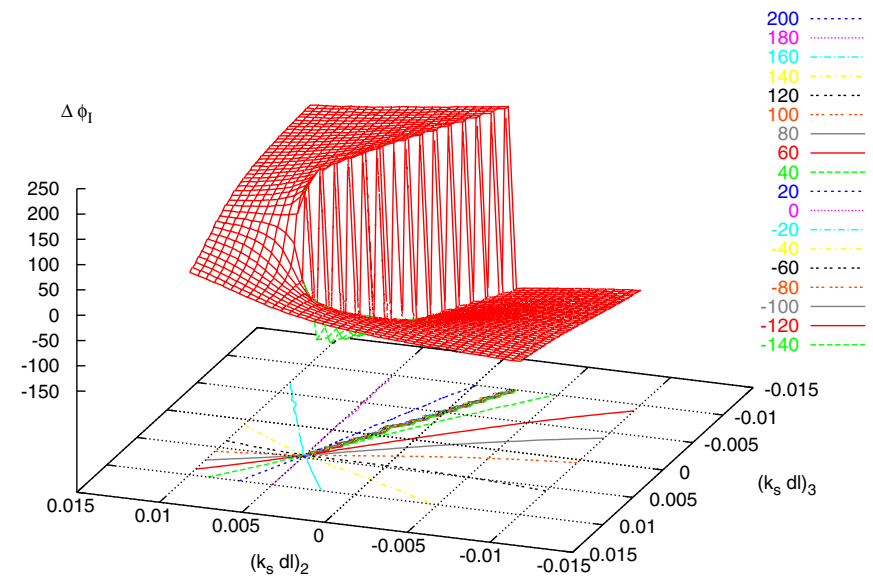

FIG. 3. (Color) Projection phase difference $\Delta \phi_{I}$ in a 2D decoupling scan. 


\section{ANALYTICAL SOLUTIONS}

In this section, we give the analytical expressions to the eigenmode projection parameters. The approach with strict matrix treatment of linear coupling is presented, followed by the Hamiltonian perturbation approach.

\section{A. Strict approach with action-angle parametrization}

With the action-angle parametrization of linear coupling $[19,20]$, a single particle's motion is represented by

$$
\left(\begin{array}{c}
x \\
x^{\prime} \\
y \\
y^{\prime}
\end{array}\right)=\mathbf{P} \cdot\left(\begin{array}{c}
\sqrt{2 J_{I}} \cos \Phi_{I} \\
-\sqrt{2 J_{I}} \sin \Phi_{I} \\
\sqrt{2 J_{I I}} \cos \Phi_{I I} \\
-\sqrt{2 J_{I I}} \sin \Phi_{I I}
\end{array}\right),
$$

where $J_{I, I I}, \Phi_{I, I I}$ are the actions and betatron phases of the two eigenmode motions, respectively. $\mathbf{P}$ can be numerically calculated from the eigenvectors of the one-turn $4 \times 4$ transfer map. It can also be expressed in the Twiss and coupling parameters defined in Edwards-Teng's parametrization:

$$
\mathbf{P}=\left(\begin{array}{cccc}
r \sqrt{\beta_{I}} & 0 & c_{11} \sqrt{\beta_{I I}}-\frac{c_{12} \alpha_{I I}}{\sqrt{\beta_{I I}}} & \frac{c_{12}}{\sqrt{\beta_{I I}}} \\
-\frac{\alpha_{I} r}{\sqrt{\beta_{I}}} & \frac{r}{\sqrt{\beta_{I}}} & c_{21} \sqrt{\beta_{I I}}-\frac{c_{22} \alpha_{I I}}{\sqrt{\beta_{I I}}} & \frac{c_{22}}{\sqrt{\beta_{I I}}} \\
-\frac{c_{12} \alpha_{I}}{\sqrt{\beta_{I}}}-c_{22} \sqrt{\beta_{I}} & \frac{c_{12}}{\sqrt{\beta_{I}}} & r \sqrt{\beta_{I I}} & 0 \\
\frac{c_{11}}{\sqrt{\beta_{I}}}+c_{21} \sqrt{\beta_{I}} & -\frac{c_{11}}{\sqrt{\beta_{I}}} & -\frac{\alpha_{I I} r}{\sqrt{\beta_{I I}}} & \frac{r}{\sqrt{\beta_{I I}}}
\end{array}\right) .
$$

Expanding Eq. (6) gives

$$
\left\{\begin{array}{l}
x=p_{11} \sqrt{2 J_{I}} \cos \Phi_{I}+p_{13} \sqrt{2 J_{I I}} \cos \Phi_{I I}-p_{14} \sqrt{2 J_{I I}} \sin \Phi_{I I} \\
y=p_{31} \sqrt{2 J_{I}} \cos \Phi_{I}-p_{32} \sqrt{2 J_{I}} \sin \Phi_{I}+p_{33} \sqrt{2 J_{I I}} \cos \Phi_{I I}
\end{array}\right.
$$

Therefore,

$$
\begin{gathered}
\left\{\begin{array}{l}
R_{I}=\frac{\sqrt{p_{31}^{2}+p_{32}^{2}}}{p_{11}} \\
R_{I I}=\frac{\sqrt{p_{13}^{2}+p_{14}^{2}}}{p_{33}},
\end{array}\right. \\
\left\{\begin{array}{l}
\Delta \phi_{I}=\arctan \left(\frac{p_{32}}{p_{31}}\right) \\
\Delta \phi_{I I}=\arctan \left(\frac{p_{14}}{p_{13}}\right),
\end{array}\right.
\end{gathered}
$$

or, in the Twiss and coupling parameters, according to Eq. (7),

$$
\begin{gathered}
\left\{\begin{array}{l}
R_{I}=\frac{\sqrt{\beta_{I} c_{22}^{2}+2 \alpha_{I} c_{22} c_{12}+\gamma_{I} c_{12}^{2}}}{r \sqrt{\beta_{I}}} \\
R_{I I}=\frac{\sqrt{\beta_{I I} c_{11}^{2}-2 \alpha_{I I} c_{11} c_{12}+\gamma_{I I} c_{12}^{2}}}{r \sqrt{\beta_{I I}}},
\end{array}\right. \\
\left\{\begin{array}{l}
\Delta \phi_{I}=\arctan \left(\frac{-c_{12}}{\alpha_{I} c_{12}+\beta_{I} c_{22}}\right) \\
\Delta \phi_{I I}=\arctan \left(\frac{c_{12}}{-\alpha_{I I} c_{12}+\beta_{I I} c_{11}}\right) .
\end{array}\right.
\end{gathered}
$$

\section{B. Perturbation approach with the Hamiltonian perturbation theory}

The Hamiltonian perturbation theory of weak linear difference coupling gives the isolated linear difference coupling Hamiltonian as [4]

$$
H_{2}=\left|C^{-}\right| \sqrt{a_{x} a_{y}} \cos \left(\Psi_{x}-\Psi_{y}+\Delta \varphi+\chi\right),
$$

where $a_{x, y}$ and $\Psi_{x, y}$ are the coupled motion's amplitudes and phases, respectively. $\Delta$ is the fractional uncoupled tune split, $\Delta=Q_{x, 0}-Q_{y, 0}-p$. The linear difference coupling coefficient $C^{-}$is defined as

$$
C^{-}=\left|C^{-}\right| e^{i \chi}=\frac{1}{2 \pi} \int_{0}^{L} \sqrt{\beta_{x} \beta_{y}} k_{s} e^{i\left[\phi_{x}-\phi_{y}-2 \pi \Delta s / L\right]} d l,
$$

where $\beta_{x, y}$ are the uncoupled betatron amplitude functions, $\phi_{x}$ and $\phi_{y}$ are the uncoupled betatron phase advances, $k_{s}$ is individual skew quadrupole's strength, $L$ is the ring circumference, and $s$ is the distance between the skew quadruple and the reference point to calculate the coupling coefficient.

After some algebraic calculations, a single particle's motion is given by [16] 


$$
\left\{\begin{array}{l}
\left.\left.x(s)=\sqrt{2 \beta_{x}}\left\{a \cos \left[\Psi_{x}+(\nu-\Delta / 2) \varphi-\chi / 2\right)\right]+b \cos \left[\Psi_{x}-(\nu+\Delta / 2) \varphi-\chi / 2\right)\right]\right\} \\
\left.\left.y(s)=\sqrt{2 \beta_{y}}\left\{c \cos \left[\Psi_{y}+(\nu+\Delta / 2) \varphi+\chi / 2\right)\right]+d \cos \left[\Psi_{y}-(\nu-\Delta / 2) \varphi+\chi / 2\right)\right]\right\}
\end{array}\right.
$$

$$
\begin{gathered}
\nu=\frac{1}{2} \sqrt{\Delta^{2}+\left|C^{-}\right|^{2}}, \\
\left\{\begin{array}{l}
\frac{c}{a}=\frac{\left|C^{-}\right|}{2 \nu+\Delta} \\
\frac{b}{d}=-\frac{\left|C^{-}\right|}{2 \nu+\Delta},
\end{array}\right.
\end{gathered}
$$

where $a, b, c$, and $d$ are decided by the initial conditions.

There are two eigentunes from Eq. (15),

$$
\left\{\begin{array}{l}
Q_{I}=Q_{x, 0}-\frac{1}{2} \Delta+\frac{1}{2} \sqrt{\Delta^{2}+\left|C^{-}\right|^{2}} \\
Q_{I I}=Q_{y, 0}+\frac{1}{2} \Delta-\frac{1}{2} \sqrt{\Delta^{2}+\left|C^{-}\right|^{2}},
\end{array}\right.
$$

with the fractional eigentune split given by

$$
\left|Q_{I}-Q_{I I}-p\right|=\sqrt{\Delta^{2}+\left|C^{-}\right|^{2}} .
$$

Comparing Eq. (15) to Eq. (1) and considering Eqs. (2)(5), one obtains

$$
\begin{gathered}
\left\{\begin{array}{l}
R_{I}=\sqrt{\frac{\beta_{y}}{\beta_{x}}} \frac{\left|C^{-}\right|}{\sqrt{\Delta^{2}+\mid C^{-1}}+\Delta} \\
R_{I I}=\sqrt{\frac{\beta_{x}}{\beta_{y}}} \frac{\left|C^{-}\right|}{\sqrt{\Delta^{2}+\left|C^{-1}\right|^{2}}+\Delta},
\end{array}\right. \\
\left\{\begin{array}{l}
\Delta \phi_{I}=\chi \\
\Delta \phi_{I I}=\pi-\chi .
\end{array}\right.
\end{gathered}
$$

\section{Interpretations}

The action-angle parametrization approach gives the strict expressions for the projection amplitude ratios and the projection phase differences. It can be used to numerically calculate the four observables. However, for weak linear difference coupling, the analytical expressions Eq. (19)-(21) from the Hamiltonian perturbation theory are more convenient and straightforward for interpreting $R_{I, I I}$ and $\Delta \phi_{I, I I}$.

According to Eq. (19), the fractional eigentune split $\left|Q_{I}-Q_{I I}-p\right|$ is determined by the fractional uncoupled tune split $\Delta$ and the amplitude of coupling coefficient $\left|C^{-}\right|$. The minimum fractional eigentune split is given by the fractional uncoupled tune split when the coupling coefficient $C^{-}$is completely compensated.

According to Eq. (20), knowing only the measured eigentune split and $R_{I}, R_{I I}$, the uncoupled tune split $\Delta$ and the coupling coefficient amplitude $\left|C^{-}\right|$can be determined. Following Eqs. (19) and (20), we obtain

$$
\left|C^{-}\right|=\frac{2 \sqrt{R_{I} R_{I I}}}{1+R_{I} R_{I I}}\left|Q_{I}-Q_{I I}-p\right|
$$

$$
\Delta=\frac{1-R_{I} R_{I I}}{1+R_{I} R_{I I}}\left(Q_{I}-Q_{I I}-p\right) .
$$

The fractional uncoupled tunes $Q_{x, y, 0}$ are

$$
Q_{x, 0}=\frac{Q_{I}+Q_{I I}}{2}+\frac{\Delta}{2},
$$

$$
Q_{y, 0}=\frac{Q_{I}+Q_{I I}}{2}-\frac{\Delta}{2} .
$$

Here, we assumed that the horizontal fractional tune is higher than the vertical fractional tune.

According to Eq. (21), the phase difference of the coupling coefficient $C^{-}$is given by $\Delta \phi_{I}$. From Eq. (14), the phase $\chi$ of the coupling coefficient is determined only by the strength combination ratios of the skew quadrupoles. Therefore if $\Delta \phi_{I}$ can be measured, then the right decoupling direction is known.

In summary, $R_{I} R_{I I}$ together with the eigentune split $\left|Q_{I}-Q_{I I}-p\right|$ determines the residual coupling coefficient's amplitude, while $\Delta \phi_{I}$ reveals the residual coupling coefficient's phase.

\section{APPLICATION TO THE RHIC RINGS}

In the following paragraphs, the eigenmode projection parameters are obtained using the RHIC PLL tune meter. A detailed description of the PLL setup for this measurement are given in our other paper, which also presents the successful use of decoupling and tune feedbacks on the RHIC ramps [21].

\section{A. Global decoupling scheme in the RHIC}

Each RHIC ring has three correction skew quadrupole families, F1, F2, and F3. The contributions to the coupling coefficient from each skew quadrupole in one family are almost the same due to the sixfold lattice structure. The coupling contributions from these three skew quadrupole families are about $120^{\circ}$ apart when they are powered with the same strength and proper polarities. In our study, we combined F1 and F3 with the same strength to produce a family F13 whose contribution to the total coupling coefficient is approximately orthogonal to that from family F2.

Knowing the coupling coefficient $C^{-}$from the measurements of the eigenmode projection parameters, the global decoupling strengths for these two orthogonal families can be calculated using the accelerator optics model,

$$
\begin{array}{r}
\Delta\left(k_{s} d l\right)_{F 2}=-\left|C^{-}\right| \cos \left(\chi-\phi_{F 2}\right) * k_{F 2}, \\
\Delta\left(k_{s} d l\right)_{F 13}=-\left|C^{-}\right| \cos \left(\chi-\phi_{F 13}\right) * k_{F 13},
\end{array}
$$


where $\left|C^{-}\right|$and $\chi$ are the residual coupling coefficient's amplitude and phase measured at the observation point. $\phi_{F 2}$ and $\phi_{F 13}$ are the angles contributed to the global coupling coefficient at the observation point by families $\mathrm{F} 2$ and $\mathrm{F} 13$, respectively, while $k_{F 2}$ and $k_{F 13}$ are the scaling factors between the skew quadrupole strengths and the coupling coefficient for families F2 and F13, respectively. All of them can be obtained based on the ideal optics model.

\section{B. PLL I/Q data processing}

The four eigenmode projections onto the $x$ and $y$ axes are continuously measured through a phase-synchronous I/ Q demodulation in the PLL tune measurement system. If the eigentunes are well tracked, the phases $\phi_{I, x}$ and $\phi_{I I, y}$ in Eq. (1) from PLL tune meter are close to zero. Actually, for each projection in Eq. (1), data sets of $\left(I_{i, z}, Q_{i, z}\right)$ are delivered from the PLL system at about $300 \mathrm{~Hz}$.

The projection amplitude and phase for one projection is given by

$$
\begin{gathered}
A_{i, z}=\sqrt{I_{i, z}^{2}+Q_{i, z}^{2}}, \\
\phi_{i, z}=\arctan 2\left(Q_{i, z}, I_{i, z}\right) .
\end{gathered}
$$

Knowing $A_{i, z}$ and $\phi_{i, z}$, according to Eqs. (2)-(5), the eigenmode projection parameters are calculated.

\section{A beam experiment test example}

The measurement of the eigenmode projection amplitude ratio was verified in the RHIC 2005 run with the $245 \mathrm{MHz}$ PLL system reported in [15]. Knowing only $R_{I} R_{I I}$ and the eigentune split $\left|Q_{I}-Q_{I I}-p\right|$, we can calculate the residual coupling coefficient's amplitude $\left|C^{-}\right|$, the uncoupled tune split $\Delta$, and the uncoupled tunes $Q_{x, y, 0}$.

In the startup of the RHIC 2006 run, with the base-band PLL system [13], the measurement of the projection phase was confirmed. Then, with the measurements of the four eigenmode projection parameters, together with the fractional eigentune split, both the global coupling's amplitude and phase are determined and global decoupling is possible.

In the following, we present one example of a beam experiment. We first introduced $\Delta(k s d l)_{2}=-2 \times$ $10^{-4} \mathrm{~m}^{-1}$ into a well decoupled RHIC Blue ring at injection. The left part in the top plot of Fig. 4 shows that the extra coupling source pushed the eigentunes farther, while the left part in the bottom plot of Fig. 4 shows the required decoupling strengths were $\Delta(k s d l)_{2}=7 \times 10^{-5} \mathrm{~m}^{-1}$, $\Delta(k s d l)_{1,3}=1 \times 10^{-4} \mathrm{~m}^{-1}$. These strengths were calculated based on Eqs. (28) and (29) with the coupling coefficient through the measurements of eigenmode projection parameters. When we set these required coupling correc-
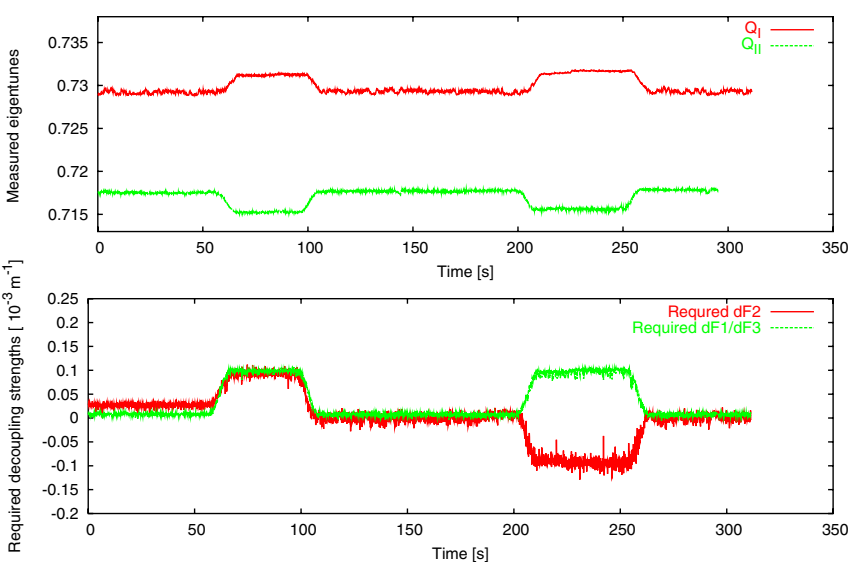

FIG. 4. (Color) The measured eigentunes and the required coupling correction strengths.

tion strengths, the eigentunes moved back and the required decoupling strengths became almost zero.

The right part of Fig. 4 shows the result when we repeated this test but with different initial coupling sources. We introduced $\Delta(k s d l)_{3}=-2 \times 10^{-4} \mathrm{~m}^{-1}$ into the above well decoupled accelerator. The required decoupling strengths were then $\Delta(k s d l)_{2}=-1 \times$ $10^{-4} \mathrm{~m}^{-1}, \quad \Delta(k s d l)_{1,3}=1 \times 10^{-4} \mathrm{~m}^{-1}$. After setting them, the ring returned to the globally uncoupled state.

The top plot of Fig. 5 shows the measured fractional eigentunes $Q_{I, I I}$ and the calculated fraction uncoupled tunes $Q_{x, y, 0}$ during these tests. It reveals that the uncoupled tunes were almost kept constant when changing the settings of the skew quadrupole strengths. When the accelerator is globally decoupled, the eigentunes and the uncoupled set tunes merged. This can be interpreted according to Eq. (18) and (19). The bottom plot of Fig. 5 shows the fractional eigentune split $\left|Q_{I}-Q_{I I}-p\right|$, the calculated uncoupled tune split $\left|Q_{x, 0}-Q_{y, 0}-p\right|$, and the coupling coefficient's $\left|C^{-}\right|$in the above tests.
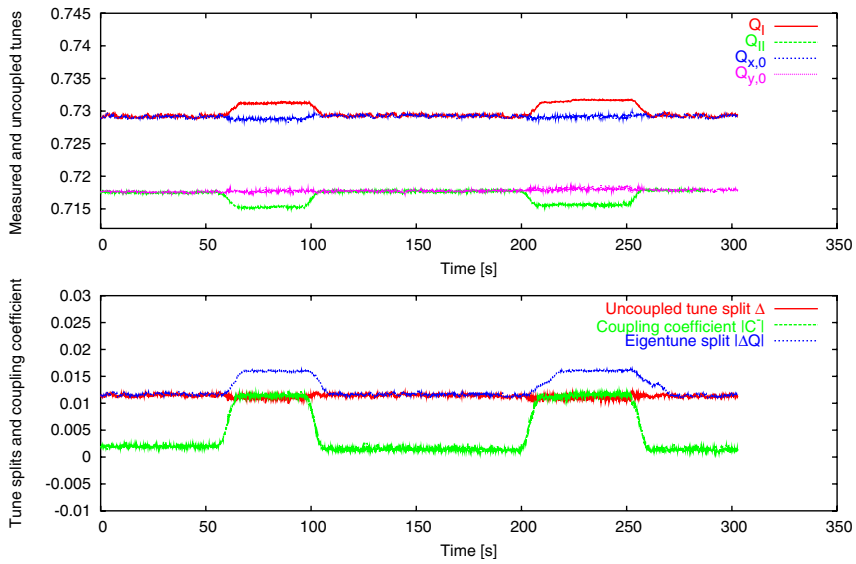

FIG. 5. (Color) The fractional tune splits and the coupling coefficient. 


\section{DISCUSSION}

The projection phase, $\Delta \phi_{I}$, plays an important role in correcting the global coupling. Its measurement is the basis of the global decoupling feedback, as it reveals the right strength combination ratios of the existing correction skew quadrupoles to compensate the residual coupling coefficient. $R_{I} R_{I I}$ determines the decoupling depth.

In the RHIC beam experiment, we assumed two orthogonal skew quadrupole families F2 and F13. For correction families which are not strictly orthogonal, iterations of the above corrections may be needed. However, having such correction families does not greatly affect the operation of the decoupling feedback.

The above analytical solutions are based on a single particle's free oscillation or coherent driven oscillation. Thus, they are also suitable for global decoupling with turn-by-turn dual-plane beam position monitor data.

In [22], a Hamiltonian approximation approach to the driven beam's response was derived through the beam transfer function. It also showed that the response of the vertical phase difference due to coherent driving in the horizontal plane gives the coupling coefficient's phase.

\section{CONCLUSION}

We presented a new technique to continuously measure and compensate the global difference coupling coefficient via the continuous measurement of eigenmode projection parameters, using a high resolution phase-locked-loop (PLL) tune meter. The analytical interpretations to the four eigenmode projection parameters are obtained with both strict matrix treatment and Hamiltonian perturbation theory of linear coupling. Based on measurements of the eigenmode projection parameters, the complex global coupling coefficient can be fully determined and compensated. This technique was successfully demonstrated in the RHIC 2006 run and yielded a global decoupling feedback along with a robust tune feedback.

\section{ACKNOWLEDGMENTS}

We would like to thank A. Chao (SLAC), W. Fischer, V. Lebedev (FNAL), V. Litvinenko, F. Pilat, V. Ptitsyn, T. Roser, R. Talman (Cornell), and D. Trbojevic for discussions and help, as well as the RHIC AP group colleagues and the RHIC operation staffs for their support during the beam experiments. This work is supported by U.S. DOE under Contract No. DE-AC02-98CH10886 and the U.S. LHC Accelerator Research Program (LARP).

[1] S. Scoch, CERN Report No. 57-20, 1957 (unpublished).

[2] G. Guignard, CERN Report No. 76-06, 1976.

[3] G. Guignard, Phys. Rev. E 51, 6104 (1995).

[4] H. Wiedemann, Particle Accelerator Physics II, Nonlinear and Higher-Order Beam Dynamics (Springer-Verlag, Berlin, 1995).

[5] D. Rubin, in Handbook of Accelerator Physics and Engineering, edited by A.W. Chao and M. Tigner (World Scientific, Singapore, 1999).

[6] Y. Luo et al., BNL C-AD AP Note 165, 2004 (unpublished).

[7] Y. Luo et al., Phys. Rev. ST Accel. Beams 8, 014001 (2005).

[8] Y. Luo et al., Phys. Rev. ST Accel. Beams 8, 074002 (2005).

[9] Y. Luo et al., in Proceedings of the 2005 Particle Accelerator Conference, Knoxville, Tennessee, p. 3182.

[10] P. Cameron et al., in Proceedings of the 2004 European Particle Accelerator Conference, Lucerne, Switzerland, p. 524.

[11] P. Cameron et al., in Proceedings of the 2004 European Particle Accelerator Conference, Lucerne, Switzerland, p. 1294.

[12] P. Cameron et al., RHIC Third Generation PLL Tune Measurement System, PAC 2003.

[13] http://www.agsrhichome.bnl.gov/LARP/ 050404_Tune_Feedback/

[14] R. Jones et al., in Proceedings of the 7th European Workshop on Beam Diagnostics and Instrumentation for Particle Accelerators, DIPAC'05, Lyon, France, 2005.

[15] Y. Luo et al., in Proceedings of the 2005 Particle Accelerator Conference, Knoxville, Tennessee, p. 3182.

[16] Y. Luo et al., BNL C-AD/AP/174, 2004 (unpublished).

[17] Y. Luo, BNL CAD/AP/214, 2005 (unpublished).

[18] Y. Luo, BNL C-AD/AP/187, 2005 (unpublished).

[19] Y. Luo, Phys. Rev. ST Accel. Beams 7, 124001 (2004).

[20] Y. Luo et al., Phys. Rev. ST Accel. Beams 8, 084001 (2005).

[21] P. Cameron et al., Phys. Rev. ST Accel. Beams 9, 122801 (2006).

[22] J.P. Koutchouk, in Proceedings of the XIth International Conference on High Energy Accelerators, CERN, Geneva, 1980. 